\title{
Near-Infrared-Activated Fluorescence Resonance Energy Transfer- Based Nanocomposite to Sense MMP2-Overexpressing Oral Cancer Cells
}

Yung-Chieh Chan ${ }^{\dagger}$ Ming-Hsien Chan, ${ }^{\ddagger}$ Chieh-Wei Chen, ${ }^{\ddagger}$ Ru-Shi Liu, ${ }^{*}, \dagger,+, \#, \perp_{\odot}$ Michael Hsiao, ${ }^{*}, \dagger, \|_{\odot}$ and Din Ping Tsai*, $\S_{,}, \mathbb{C}^{-}$

${ }^{\dagger}$ Genomics Research Center, Academia Sinica, Taipei, Taiwan

${ }^{\ddagger}$ Department of Chemistry, National Taiwan University, Taipei 106, Taiwan

${ }^{\S}$ Research Center for Applied Sciences, Academia Sinica, Taipei 115, Taiwan

"Department of Biochemistry, College of Medicine, Kaohsiung Medical University, Kaohsiung, Taiwan

${ }^{\perp}$ Department of Mechanical Engineering and Graduate Institute of Manufacturing Technology, National Taipei University of Technology, Taipei 106, Taiwan

\# Department of Physics, National Taiwan University, Taipei 106, Taiwan

\section{Supporting Information}

\begin{abstract}
The matrix metalloproteinases (MMPs) are well-known mediators that are activated in tumor progression. MMP2 is a kind of gelatinase in extracellular matrix remodeling and cancer metastasis processes. MMP2 secretion increased in many types of cancer diseases, and its abnormal expression is associated with a poor prognosis. We fabricated a nanocomposite that sensed MMP2 expression by a red and blue light change. This nanocomposite consisted of an upconversion nanoparticle (UCNP), MMP2sensitive peptide, and $\mathrm{CuInS}_{2} / \mathrm{ZnS}$ quantum dot (CIS/ZnS QD). An UCNP is composed of $\mathrm{NaYF}_{4}: \mathrm{Tm} / \mathrm{Yb} @ \mathrm{NaYF}_{4}: \mathrm{Nd} / \mathrm{Yb}$, which has multiple emissions at UV/blue-visible wavelengths under $808 \mathrm{~nm}$ laser excitation. The conjugated CIS/ZnS QD showed the redvisible fluorescence though the FRET process. The two fluorophores were connected by a MMP2-sensitive peptide to form a novel MMP2 biosensor, named UCNP@p-QD. UCNP@p-QD was highly biocompatible according to cell viability assay. The FRETbased biosensor was employed in the MMP2 determination in vitro and in vivo.

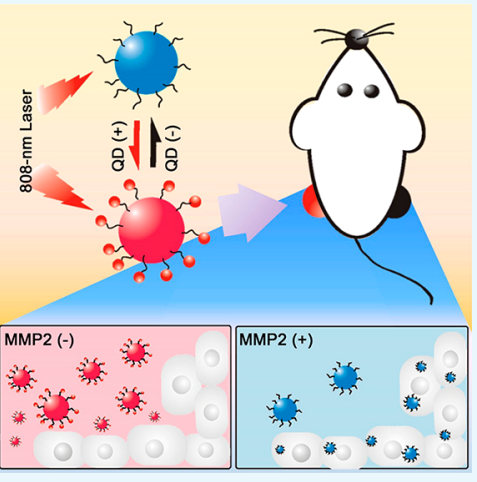
Furthermore, it was administrated into the tumor-bearing mouse to check MMP2 expression. UCNP@p-QD could be a promising tool for biological study and biomedical application. In this study, we demonstrated that the CIS/ZnS QD improved the upconversion intensity through a near-infrared-induced FRET process. This nanocomposite has the advantage of light penetration, excellent biocompatibility, and high sensitivity to sense MMP2. The nearinfrared-induced composites are a potential inspiration for use in biomedical applications.
\end{abstract}

\section{INTRODUCTION}

Cancer metastasis is one of the deadliest symptoms in cancerrelated diseases. The extracellular matrix (ECM) remodeling occurs during cancer metastasis, which assists tumor growth and cancer cell dissemination. ${ }^{1,2}$ The matrix metalloproteinase2 (MMP2) family plays the pivotal role in ECM remodeling, leading to cancer cell invasion. ${ }^{3}$ In general, the active MMP2 is responsible for degrading type IV collagen. However, in tumorigenesis, abnormal MMP2 expression is associated with cancer cell motility and angiogenesis. ${ }^{4-6} \mathrm{MMP} 2$ overexpression significantly promoted the lymphatic metastasis in oral cancer patients. $^{7}$ Administration of MMP2 microRNA could be an adjuvant to inhibit mouse glioma in radiotherapy. ${ }^{8}$ An MMP2sensitive paclitaxel prodrug could maintain anticancer activity by increasing permeability, cancer cell targeting, and retention effect. ${ }^{9}$ A self-assembly nanodrug that conjugated an amphiphilic molecule and antitumor drug by an MMP2- sensitive peptide was suggested to have the potential in clinical translation to increase the biocompatibility and reduce the side effects of drug accumulation. ${ }^{10}$ A photosensitizer therapeutic agent was conjugated with MMP2-cleavable peptide and then modified with PEG to form a self-assembled and redoxresponsive system for fluorescent monitoring and photodynamic therapy. ${ }^{11}$ The pterostilbene compound that blocked the MMP2-induced signal pathway suppressed the oral cancer cell invasion. ${ }^{12}$ The MMP2 antibody labeled with the Cy5 probe could be used in in vivo animal imaging. ${ }^{13}$ Thus, an MMP2-senstive probe has potential in cancer metastasis theranostics.

Received: October 6, 2017

Accepted: January 26, 2018

Published: February 8, 2018 
Lanthanide-doped upconversion nanoparticles (UCNPs) have been extensively investigated according to its unique photochemistry. ${ }^{14}$ By NIR excitation, UCNP emits an antistoke type and split emissions at UV/visible spectra. Upconversion luminescence is triggered by sensitizer $\left(\mathrm{Yb}^{3+}\right)$ and emitters $\left(\mathrm{Er}^{3+}\right.$ and $\left.\mathrm{Tm}^{3+}\right)$ under $980 \mathrm{~nm}$ irradiation. ${ }^{15}$ Moreover, a core/ shell UCNP emits upconversion luminescence by an $808 \mathrm{~nm}$ laser. $^{16}$ After PEG coating, UCNP-based nanomaterial can catch protein for bioanalytical applications. ${ }^{17}$ The core/shell UCNP coated with iron and organic compounds shows a tumor-specific targeting ability. ${ }^{18}$ Yao et al. showed that an UCNP-assisted liposome drug delivery system could overcome drug resistance and release drug by a NIR trigger. ${ }^{19}$ A CdTeS QD has been shown to have potential for biosensor detection of MMP2 secretion in vitro and in vivo. ${ }^{20}$ The copper indium sulfide (CIS) QD is more biocompatible than other QDs. ${ }^{21}$ CIS QD is a I-III-V ternary semiconductor. ${ }^{22}$ CIS QD has the dramatic advantages in tunable photoluminescence and high quantum yield. However, the hydrophobic property limited its bioapplication. The cytotoxicity of CIS/ZnS QD with chitosan coating has been determined by long-term incubation with $C$. elegans. $^{23}$ The silica-encapsulated CIS/ZnS QD remains an excellent optical property with hydrophilic ability. ${ }^{24}$

Optical imaging is a considerable strategy in medical diagnostics because of its noninvasiveness, sensitivity, and convenience. However, the tissue penetration is an Achilles' heel for the optical diagnostic tool. ${ }^{25}$ The penetration depth increased depending on increasing wavelength, except the 900$1000 \mathrm{~nm} .{ }^{26}$ The penetration of laser light at 808 and $980 \mathrm{~nm}$ reached 3.4 and $2.2 \mathrm{~cm}$, respectively. ${ }^{27}$ The decrease of penetration depth at $900-1000 \mathrm{~nm}$ was due to water absorption, suggesting that the $808 \mathrm{~nm}$ irradiation has potential in biomedical application. ${ }^{28}$ This study demonstrated a fluorescence resonance energy transfer-based (FRET) nanocomposite consisting of UCNP, MMP2-sensitive peptide, and CIS/ZnS QD, named UCNP@p-QD. The core/shell UCNP contributed NIR-induced upconversion energy to CIS/ZnS QD with red-visible emission. We showed that UCNP@p-QD was a biocompatible nanocomposite, and it responded to the MMP2 protein in oral cancer cells and the animal tumor model.

\section{RESULTS AND DISCUSSION}

In this study, we developed a FRET-based nanocomposite that detected MMP2 expression in vivo. This nanocomposite consists of UCNP and CIS/ZnS QDs, which were linked by an MMP2-sensitive peptide (Figure 1a). UCNP@p-QD has FRET-induced red fluorescence by $808 \mathrm{~nm}$ irradiation. This nanocomposite revealed the blue fluorescence in the presence of MMP2. At first, we fabricated a core/shell type $\mathrm{NaYF}_{4}: \mathrm{Yb}$ / $\mathrm{Tm} @ \mathrm{NaYF}_{4}: \mathrm{Yb} / \mathrm{Nd} \mathrm{UCNP}$. The core was $\mathrm{NaYF}_{4}: \mathrm{Yb}^{3+} / \mathrm{Tm}^{3+}$ that emitted multifluorescence under NIR irradiation. The data of the hexagonal $\beta$-NaYF4 database (JCPDS 16-0334) were compared with $\mathrm{NaYF}_{4}: \mathrm{Yb}, \mathrm{Tm}$ and $\mathrm{NaYF}_{4}: \mathrm{Yb}, \mathrm{Tm}$. The diffraction peaks were similar to those of the standard peaks (Figure 1b). UCNPs were uniform in aqueous solution of which the diameter was approximately $20 \mathrm{~nm}$ (Figure 1c). Doped with $\mathrm{Yb}^{3+}$ and $\mathrm{Nd}^{3+}$, the core/shell type, which formed a hexagonal structure, was in response to $808 \mathrm{~nm}$ excitation. In order to graft the MMP2-sensitive peptide, UCNP was modified with primary amine on the $\mathrm{SiO}_{2}$ layer. ${ }^{29}$ The $\mathrm{SiO}_{2}$ absorption was revealed at $1100 \mathrm{~cm}^{-1}$ compared to ligand-free UCNP (Figure S1a, Supporting Information). The absorption of UCNP@p at the Si-O-Si $\left(1100 \mathrm{~cm}^{-1}\right), \mathrm{N}-\mathrm{C}=\mathrm{O}(1660$ (a)

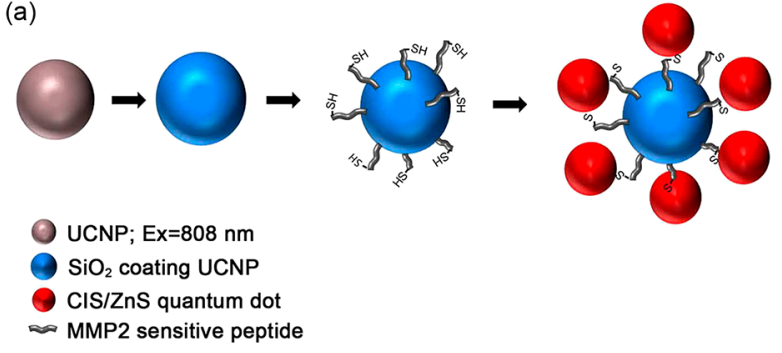

(b)

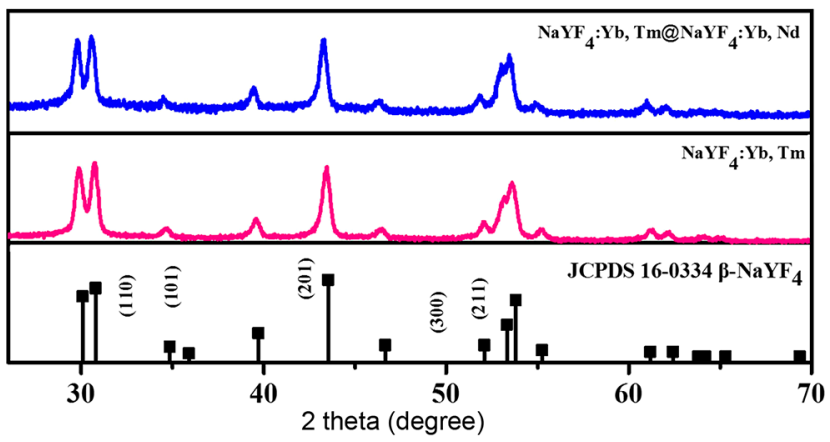

(c)

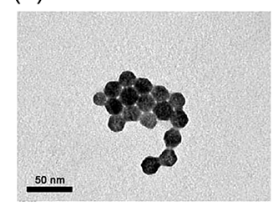

(f)

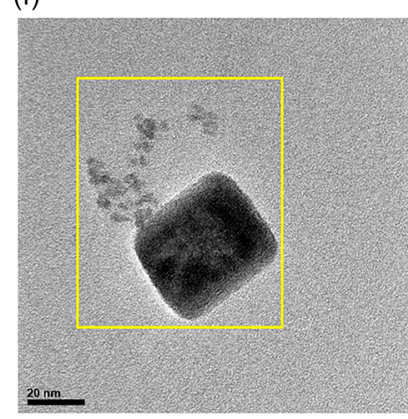

(d)

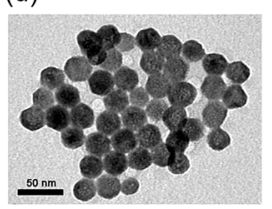

(e)

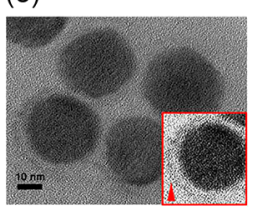

(g)

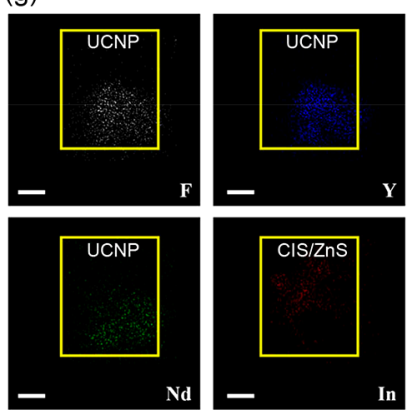

Figure 1. Preparation and characterization of UCNP@p-QD. (a) Schematic showing the fabrication strategy of UCNP@p-QD. UCNP was coated with $\mathrm{SiO}_{2}$ and decorated with MMP2-sensitive peptide. CIS/ZnS QD conjugated onto the peptide on the UCNP. (b) The Xray diffractions of NaYF4:Yb/Tm@NaYF4:Yb/Nd and NaYF4:Yb/ Tm were compared with the $\beta$-NaYF4 database (JCPDS 16-0334). (c) The uniform UCNP was observed by TEM. The scale bar represents $50 \mathrm{~nm}$. (d) The UCNP was coated with $\mathrm{SiO}_{2}$. The scale bar represents $50 \mathrm{~nm}$. (e) High-resolution TEM showed the QD could be conjugated onto UCNP. The arrow indicated the QD (inset). The scale bar represents $10 \mathrm{~nm}$. (f) High-resolution TEM image and the elemental mapping (g) of UCNP (F, Y, Nd) and CIS/ZnS QD (In). The scale bar represents $20 \mathrm{~nm}$. The yellow rectangular frame indicated the same proportions in EDS mapping analysis.

$\left.\mathrm{cm}^{-1}\right)$, and $\mathrm{C}-\mathrm{O}\left(1100-1350 \mathrm{~cm}^{-1}\right)$ corresponded with the standard curve of UCNP and peptide. The $\mathrm{SiO}_{2}$-coated UCNP showed the size of $25 \mathrm{~nm} \pm 1 \mathrm{~nm}$ (Figure 1d). The N-terminal part from UCNP embedded a cysteine residue, to which the QD conjugated through disulfide linkage. ${ }^{30} \mathrm{X}$-ray diffraction (XRD) of the CIS/ZnS showed the major peaks corresponding to the standard diffraction patterns of CIS crystal (JCPDS No. 
(a)

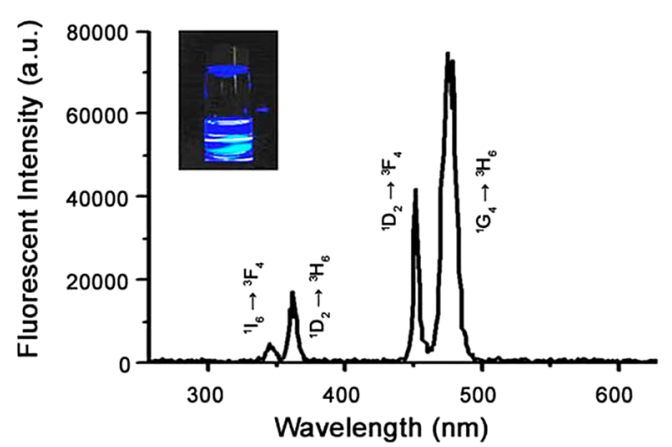

(c)

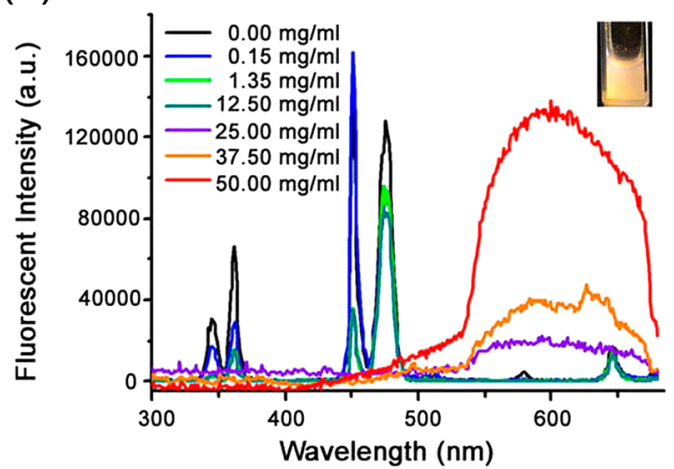

(b)

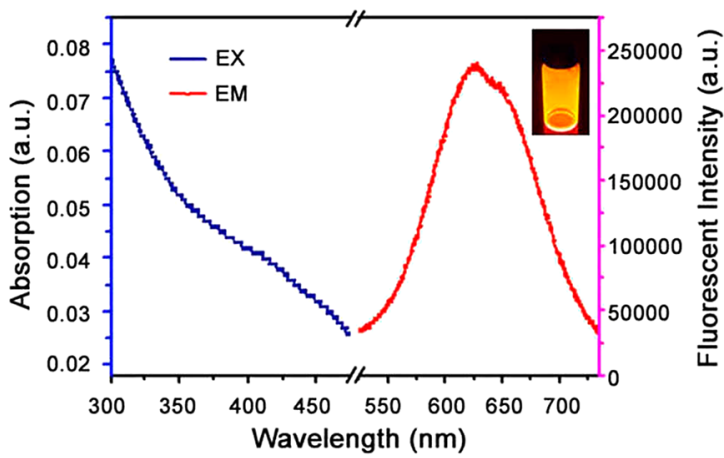

(d)

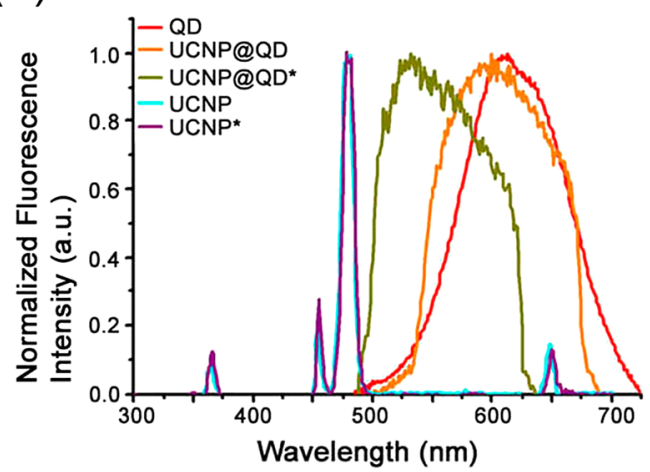

Figure 2. Optical properties of UCNP, CIS/ZnS QD, and UCNP@p-QD. (a) Photoluminescence analysis (PL) of UCNP. Inset showed upconversion fluorescence under $808 \mathrm{~nm}$ excitation. (b) Ultraviolet-visible spectroscopy of CIS/ZnS QD. Blue fluctuation showed QD absorption, and red fluctuation was the emission region of the QD. Inset represented fluorescence from the QD. (c) PL spectra of conjugating with different QD concentration. UCNP@p-QD showed the FRET fluorescence under $808 \mathrm{~nm}$ irradiation (inset). (d) The normalized PL spectra of QD, UCNP@pQD, UCNP@p-QD*, UCNP, and UCNP*. The attached symbol $\left(^{*}\right)$ indicated UCNP@p-QD decorated with PEG polymers. PL showed the emissions under $808 \mathrm{~nm}$ excitation except for unconjugated QD (360 $\mathrm{nm}$ excitation).

85-1175) and ZnS crystal (JCPDS No. 77-2100) at $27^{\circ}, 47^{\circ}$, and $55^{\circ}$ (Figure S1b, Supporting Information). The CIS/ZnS QD is a stable and biocompatible nanoparticle that is appropriate to bioapplication. ${ }^{23}$ The distance between QD and UCNP was close to $10 \mathrm{~nm}$ that was FRET allowed between donor and acceptor. UCNP@p-QD was approximate to $30 \mathrm{~nm}$ in size (Figure 1e). CIS $/ \mathrm{ZnS}$ QD with size of $3.5 \mathrm{~nm}$ $\pm 0.2 \mathrm{~nm}$ was surrounded around the UCNP@p (inset, Figure 1e). The EDS analysis indicated that QDs were bound tightly with UCNP (Figure S1c, Supporting Information), in which the elements of QDs, such as In, $\mathrm{S}$, and $\mathrm{Cu}$, were located next to the elemental cluster within $\mathrm{Nd}$ and $\mathrm{Yb}$. Moreover, the elements of UCNP and CIS/ZnS QD were identified from the conjugated structure using elemental mapping (Figure 1f). According to the elemental distribution, the fluorine $(F)$, yttrium $(\mathrm{Y})$, and neodymium $(\mathrm{Nd})$ elements of UCNP appeared in the core, while the indium (In) of CIS/ZnS QD was distributed in the periphery (Figure 1g).

UCNP showed antistoke shift fluorescence under $808 \mathrm{~nm}$ laser irradiation (Figure 2a). The $\mathrm{Nd}^{3+}$ ion was a sensitizer, in which the electron was excited from the ground state to the excited state. ${ }^{31}$ Meanwhile, a nonradiative energy transfers to the excited level ${ }^{2} \mathrm{~F}_{5 / 2}$ of $\mathrm{Yb}^{3+}$. The excited state of $\mathrm{Tm}^{3+}$ has four energy relaxations at wavelengths of $340,360,450$, and $475 \mathrm{~nm} .340$ and $360 \mathrm{~nm}$ were due to the electronic transition ${ }^{1} \mathrm{I}_{6} \rightarrow{ }^{3} \mathrm{~F}_{4}$ and ${ }^{1} \mathrm{D}_{2} \rightarrow{ }^{3} \mathrm{H}_{6}$. The blue-visible light was contributed by transitions of ${ }^{1} \mathrm{D}_{2} \rightarrow{ }^{3} \mathrm{~F}_{4}$ and ${ }^{1} \mathrm{G}_{4} \rightarrow{ }^{3} \mathrm{H}_{6}$. QD has a broad absorption below $500 \mathrm{~nm}$ and has red fluorescence emission at $625 \mathrm{~nm}$ (Figure 2b). QD showed an orange emission under UV irradiation (inset, Figure 2b). To develop not only high penetration of excitation source but also a red shift, the fluorophore is desired for clinical use. UCNP acted as a donor that was excited by $808 \mathrm{~nm}$ irradiation. The acceptor CIS/ZnS QD that was excited by the upconversion fluorescence was a red fluorophore. The FRET process was manifested by the fact that the fluorescence intensity of the UV and visible-light spectrum showed a gradual decrease depending on increasing QD concentration (Figure 2c). To delineate the FRET dynamic change, we focused on the wavelengths at 475 and $600 \mathrm{~nm}$, to which UCNP and QD contributed, respectively (Figure S2a, Supporting Information). The red fluorescence revealed at $25 \mathrm{mg} / \mathrm{mL}$ of $\mathrm{QD}$ conjugation implied a threshold of FRET-induced red fluorescence. Furthermore, the zetapotential analysis indicated that the positive charge of UCNP@ $\mathrm{p}$ decreased by the increasing QDs (Figure S2b, Supporting Information). Since the CIS/ZnS QD was hydrophobic, it dissolved in organic solvent rather than in $\mathrm{H}_{2} \mathrm{O}$. We then modified the UCNP@p-QD synthesis process by PEGlyation onto the surface of the QD. ${ }^{32}$ PEGlyation was carried out by

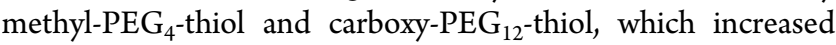
the hydrophilicity and minimized nonspecific binding (Figure S2c, Supporting Information). The 2853 and $2925 \mathrm{~cm}^{-1}$ were from the methylene-stretching group of PEG. The emission of UCNP@p-QD with $808 \mathrm{~nm}$ irradiation is similar to QD under 
(a)

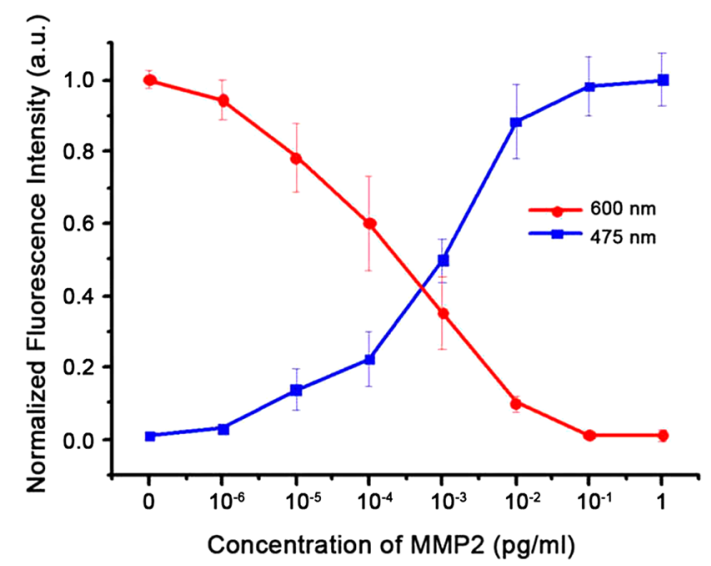

(b)

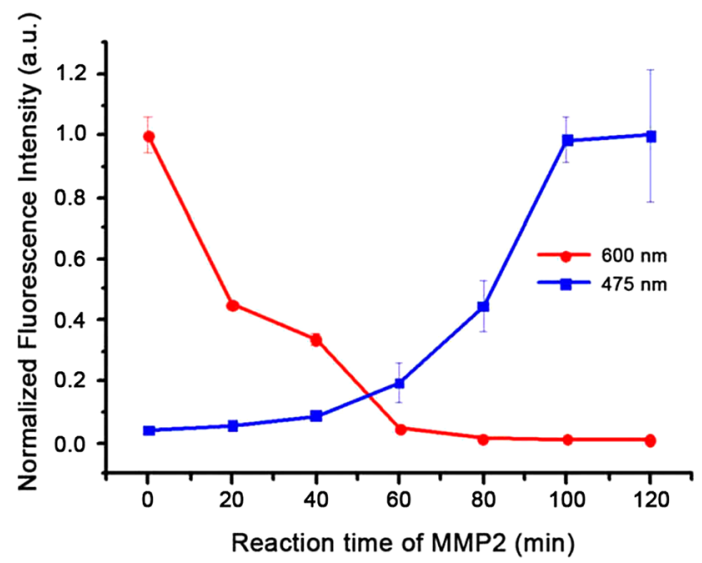

Figure 3. Photoluminescence analysis showed the FRET change of UCNP@p-QD in the presence of rhMMP2 under $808 \mathrm{~nm}$ irradiation. The blue line indicated the visible fluorescence of UCNP at $475 \mathrm{~nm}$, and the red line indicated the FRET-induced florescence at $600 \mathrm{~nm}$. (a) UCNP@p-QD was incubated with different concentrations of rhMMP2. The concentration of rhMMP2 was $0,10^{-6}, 10^{-5}, 10^{-4}, 10^{-3}, 10^{-2}, 10^{-1}$, and $1 \mathrm{pg} / \mathrm{mL}$, respectively. (b) The time-lapse PL analysis showed that UCNP@p-QD was incubated with $1 \mathrm{pg} / \mathrm{mL}$ of rhMMP2. The checkpoint was 20, 40, 60, 80,100 , and $120 \mathrm{~min}$.

UV excitation. The PEGlyated UCNP@p-QD has orange fluorescence at wavelength of $550 \mathrm{~nm}$ (Figure 2d). Obviously, there is about a $100 \mathrm{~nm}$ blue shift after PEGlyation. Park et al. suggested that cation exchange could promote a blue shift in emission. ${ }^{33}$ Ryu et al. suggested that the addition of surfactant could contribute to the blue shift. ${ }^{34}$ In our experimental result, the surface reconstruction of UCNP@p-QD led to an apparent blue-shift emission. The blue-shift mechanism should be further demonstrated.

We soaked $10 \mu \mathrm{g}$ of UCNP@QD into $1 \mathrm{~mL}$ of PBS at different $\mathrm{pH}$ values of $2,4,7,9$, and 12, respectively (Figure S3a, Supporting Information). Dynamic light scattering (DLS) showed that the hydration radii between $\mathrm{pH} 4$ and $\mathrm{pH} 9$ were not obviously changed, implying that UCNP@p-QD was stable in biological solution. UCNP@p-QD $(0.2 \mathrm{mg} / \mathrm{mL})$ was incubated with different concentration of MMP2 (Figure S3b, Supporting Information). The 600 and $475 \mathrm{~nm}$ emissions delineated a FRET change that originated from $Q D$ and UCNP, respectively. The fluctuations showed that the FRETinduced fluorescence $(600 \mathrm{~nm})$ gradually decreased; meanwhile, upconversion fluorescence $(475 \mathrm{~nm})$ increased depending on increasing rhMMP2 (Figure 3a). The recovery of upconversion fluorescence indicated that MMP2-sensitive peptides were digested, and then QDs were separated from UCNP. The FRET-induced and upconversion fluorescence were simultaneously detected at the interval of $10^{-5}$ to $10^{-2} \mathrm{pg} /$ $\mathrm{mL}$. In the concentration of $10^{-1} \mathrm{mg} / \mathrm{mL}$, the intensity of upconversion fluorescence reached a plateau, and the FRETinduced red fluorescence declined to the zero, indicating that the $0.2 \mathrm{mg} / \mathrm{mL}$ of UCNP@p-QD could be the effective dose. In time-dependent rhMMP2 digestion, the FRET was consistently expressed until the cleavage time reached $60 \mathrm{~min}$ (Figure S3c, Supporting Information). Not only the increase of upconversion fluorescence but also the decrease of FRETinduced fluorescence showed exponential fluctuations. The FRET-induced fluorescence severely decreased between 0 and $20 \mathrm{~min}$. The upconversion fluorescence intensity was retrieved serially along with reaction time until a plateau at $100 \mathrm{~min}$ (Figure 3b). The FRET elimination of UCNP@p-QD showed the presence of rhMMP2. This implied the UCNP@p-QD could be employed in clinical MMP2 detection such as a blood specimen.

The cell viability analysis showed that UCNP@p-QD could be biocompatible to oral cancer cells (Figure S4a, Supporting Information). The high dose of UCNP@p-QD reduced 5\% viabilities of FADU and OEC-M1. This implied that $83 \mu \mathrm{g} / \mathrm{mL}$ of dosage was safe to FADU and OEC-M1 cells rather than 250 $\mu \mathrm{g} / \mathrm{mL}$. The $808 \mathrm{~nm}$ laser showed a little phototoxicity (Figure S4b, Supporting Information). Moreover, a high dose of UCNP@p-QD showed little toxicity to Cal27 cells (Figure S4c, Supporting Information). To our knowledge, CIS/ZnS QD was safe for C. elegan. ${ }^{23}$ The cover of the QD might reduce certain cytotoxicity from UCNP@p because the increasing UCNP@p revealed toxicity (Figure S4d, Supporting Information). Thus, UCNP@p-QD was biocompatible to cells.

The extracellular UCNP@p-QD showed red emission under 808 nm irradiation. After MMP2 digestion, the UCNP@p showed blue upconversion fluorescence (Figure 4a). To detect the MMP2 in the cell model, constituted MMP2-overexpressing cells (Cal27/MMP2) and their cognate cells (Cal27/VC) were cultured for UCNP@p-QD detection. Cal27/MMP2 overexpressed the MMP2 protein not only in cytoplasm but also in the culture medium. ${ }^{35}$ The FRETinduced red fluorescence showed in Cal27/VC but also in Cal27/MMP2 (Figure 4b). The UCNP@p-QD accumulated around Cal27/VC cells, indicating there was no MMP2 expression; oppositely, the FRET-induced fluorescence was significantly reduced in Cal27/MMP2. Moreover, UCNP@pQD showed the same consequence in OEC-M1 and FADU cells (Figure S5, Supporting Information), which were MMP2null and MMP2-overexpressing cells, respectively. ${ }^{35}$ This result indicated that UCNP@p-QD could be detected in extracellular MMP2. The effective penetration is usually an obstacle for scientists to develop the photodependent biosensors, so the high penetrating infrared has attracted the attention of investigators.

In order to take an in vivo image, we refitted an optical imaging system to employ the upconversion luminescence detection (Figure S6a, Supporting Information). Under $808 \mathrm{~nm}$ irradiation, the FRET-induced fluorescence was detected on the 
(a)

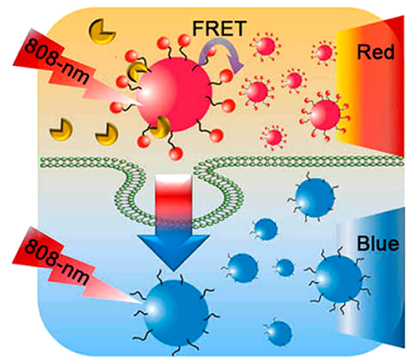

(b)

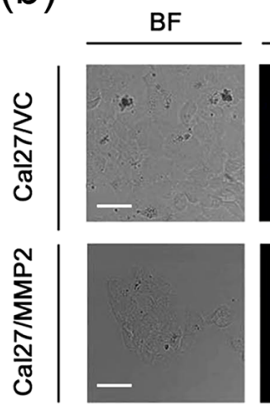

(C)
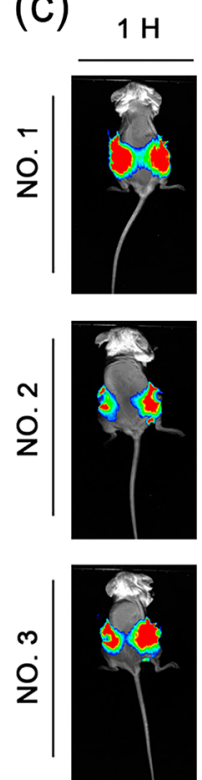
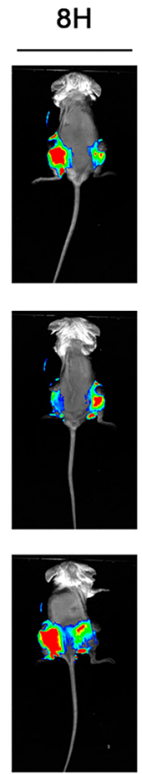
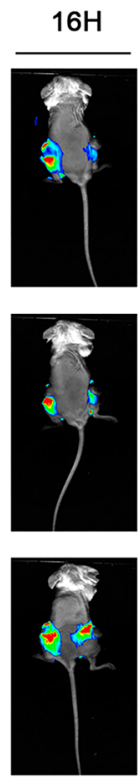
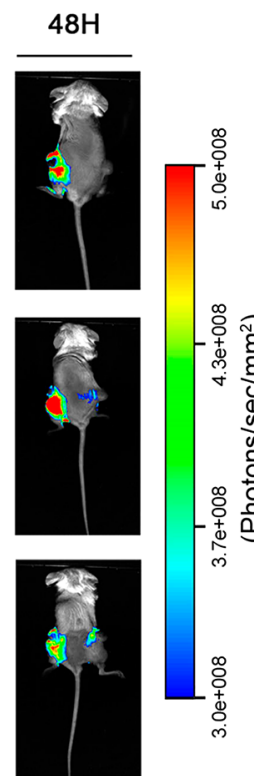
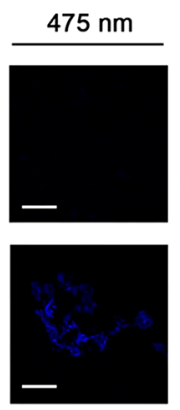
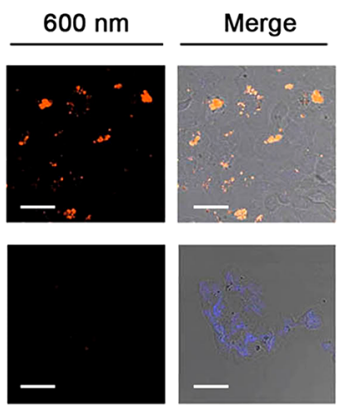

Cal 27

(d)

VC
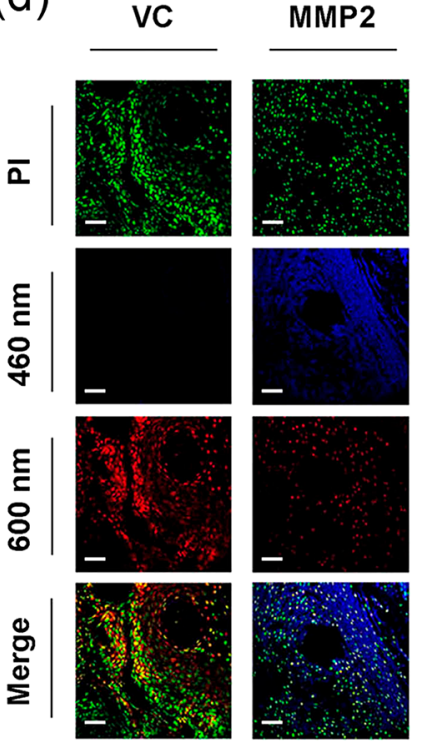

Figure 4. (a) Schematic illustration indicating the FRET change of UCNP@p-QD in the presence of MMP2. (b) In vivo MMP2 sensitivity of UCNP@p-QD was performed in Cal27/VC and Cal27/MMP2. The FRET change showed FRET-induced fluorescence (600 nm) and upconversion fluorescence $(475 \mathrm{~nm})$ were discriminately expressed by MMP2 alternation. Dynamic tracking of UCNP@p-QD performed in the mouse tumor model. The scale bar represents $50 \mu \mathrm{m}$. (c) By intratumoral injection, UCNP@p-QD was administrated into Cal27/VC (left side of mice) and Cal27/MMP2 (right side of mice) induced tumors. FRET-induced images were detected at $1 \mathrm{~h}, 8 \mathrm{~h}, 16 \mathrm{~h}$, and $48 \mathrm{~h}$ under $808 \mathrm{~nm}$ irradiation. (d) FRET change, focusing on 475 and $600 \mathrm{~nm}$, was observed from a tumor section that was stained with propidium iodide (PI). These tumor sections were prepared from NO.1 mouse. The scale bar represents $50 \mu \mathrm{m}$.

separated dorsal side within $1 \mathrm{~h}$ (Figure 4c; column $1 \mathrm{H}$ ). Distribution of UCNP@p-QD was visualized uniformly in tumor and its adjacent part. Additionally, the RFP-harbor $\mathrm{Cal27/VC}$ cell could be specifically examined on the left dorsal side (Figure S6b, Supporting Information). The FRET-induced fluorescence significantly decreased over time in Cal27/MMP2induced tumors $(1 \mathrm{H}$ to $48 \mathrm{H})$. Finally, the FRET-induced fluorescence almost disappeared from the Cal27/MMP2induced tumor part (Figure $4 \mathrm{c}$; column $48 \mathrm{H}$ ). Although there is a little FRET-induced fluorescence from Cal27/MMP2induced tumor (Figure 4c; NO.3), most UCNP@p-QDs were significantly degraded by MMP2. The harvested tumors showed the similar size and pathological feature between Cal27/VC- and Cal27/MMP2-induced tumors (Figures S6c and S6d, Supporting Information). Because the blue-visible filter is not equipped in an in vivo imaging system, the tumor sections were examined using a multiphoton microscope (Figure 4d). The UCNP@p-QDs were shown ubiquitously in the Cal27/VC tumor tissue. Oppositely, the upconversion fluorescence was detected in the Cal27/MMP2 tumor. Hence, UCNP@p-QD detected not only rhMMP2 protein in vitro but also MMP2 expression in the cell model and animal tumor imaging.

\section{CONCLUSIONS}

In summary, we fabricated a FRET-based nanocomposite comprising an $808 \mathrm{~nm}$-induced upconversion nanoparticle, the core/shell type of CIS/ZnS quantum dot, and the MMP2sensitive peptide, named UCNP@p-QD. Upconversion fluorescence from UCNP was excited by $808 \mathrm{~nm}$ laser irradiation which is known for low phototoxicity and high penetration. Consequently, the upconversion fluorescence transferred to $\mathrm{CIS} / \mathrm{ZnS} \mathrm{QD}$ and showed red fluorescence at $600 \mathrm{~nm}$. We demonstrated that NIR laser energy was transferred to FRETinduced fluorescence though an antistokes shift and then a stokes shift process. This improved the poor upconversion fluorescence of UCNP by using CIS/ZnS QDs. The in vitro experiment characterized that UCNP@p-QD was susceptible to rhMMP2 proteinase with high sensitivity and selectivity. Because the FRET-based UCNP@p-QD was biocompatible in cell viability assay, we employed UCNP@p-QD in determining MMP2 expression in the cell model. Furthermore, the 
biosensor was used to monitor the MMP2-induced tumor in an in vivo mouse tumor model. The FRET-based nanocomposite would be worthy to further investigate by replacing other specific enzyme-sensitive peptides in clinical diagnostics.

\section{MATERIALS AND METHODS}

Chemicals and Media. All chemicals were purchased from commercial suppliers without further purification. Y$\left(\mathrm{CH}_{3} \mathrm{CO}_{2}\right)_{3} \cdot \mathrm{H}_{2} \mathrm{O}, \mathrm{Yb}\left(\mathrm{CH}_{3} \mathrm{CO}_{2}\right)_{3} \cdot 4 \mathrm{H}_{2} \mathrm{O}, \mathrm{Tm}\left(\mathrm{CH}_{3} \mathrm{CO}_{2}\right)_{3} \cdot \mathrm{H}_{2} \mathrm{O}$, $\mathrm{Nd}\left(\mathrm{CH}_{3} \mathrm{CO}_{2}\right)_{3} \cdot \mathrm{H}_{2} \mathrm{O}$, octadecene (ODE, $90 \%$ ), oleic acid (OA, $90 \%)$, ammonium fluoride $\left(\mathrm{NH}_{4} \mathrm{~F}\right)$, sodium hydroxide $(\mathrm{NaOH}$, $98 \%)$, hydrochloric acid ( $\mathrm{HCl}, 37 \%)$, ethanol $\left(\mathrm{C}_{2} \mathrm{H}_{6} \mathrm{O}, 99 \%\right)$, urea powder $\left(\mathrm{NH}_{2} \mathrm{CONH}_{2}, 98 \%\right)$, 3-triethoxysilylpropylamine (APTE; 98\%), folic acid 1-dodecanethiol (DDT; $\geq 98 \%$ ), $\mathrm{N}$ hydroxysuccinimide (NHS), ethyl(dimethylaminopropyl) carbodiimide (EDC), and dimethyl sulfoxide (DMSO) were purchased from Sigma-Aldrich. Trisodium citrate $\left(\mathrm{Na}_{3} \mathrm{C}_{6} \mathrm{H}_{5} \mathrm{O}_{7}\right.$, 99\%) was purchased from Acros Organics. Cyclohexane $\left(\mathrm{C}_{6} \mathrm{H}_{12}, 99 \%\right)$ was purchased from J. T. Baker. Poly(ethylene glycol) monomethyl ether thiol (methyl-PEG 4 -thiol) and poly(ethylene glycol) carboxyl ether thiol (carboxy-PEG ${ }_{12}$ thiol) were purchased from Thermo scientific. DMEM medium, MEM medium, and RPMI medium were purchased from Invitrogen. Human recombinant MMP2 (rhMMP2) protein was purchased from Biotools (Taiwan). CIS/ZnS QD was purchased from Hopax Chemicals (Taiwan Hopax Chemicals. Mfg. Co., Ltd.).

Preparation of $\mathrm{NaYF}_{4}: \mathrm{Yb} / \mathrm{Tm} @ \mathrm{NaYF}_{4}: \mathrm{Yb} / \mathrm{Nd}$. The core particles, $\mathrm{NaYF}_{4}: \mathrm{Yb} / \mathrm{Tm}$, were synthesized by the coprecipitation method under high-temperature reaction. Amounts of 0.4 $\mathrm{mL}$ of $\mathrm{Tm}\left(\mathrm{CH}_{3} \mathrm{CO}_{2}\right)_{3} \cdot \mathrm{H}_{2} \mathrm{O}, \mathrm{Yb}\left(\mathrm{CH}_{3} \mathrm{CO}_{2}\right)_{3} \cdot 4 \mathrm{H}_{2} \mathrm{O}$, and $\mathrm{Y}$ $\left(\mathrm{CH}_{3} \mathrm{CO}_{2}\right)_{3} \cdot \mathrm{H}_{2} \mathrm{O}$ were dissolved in a mixture of $\mathrm{OA}(6 \mathrm{~mL})$ and ODE $(14 \mathrm{~mL})$ at $120{ }^{\circ} \mathrm{C}$ for $30 \mathrm{~min}$. The dissolved salts immediately cooled to room temperature, and methanol solution with $\mathrm{NH}_{4} \mathrm{~F}(0.15 \mathrm{~g})$ and $\mathrm{NaOH}(0.1 \mathrm{~g})$ was added with vigorous stirring. The reaction mixture was heated to 290 ${ }^{\circ} \mathrm{C}$ for $2 \mathrm{~h}$ and was returned to room temperature. Nanoparticle precipates were washed with cyclohexane by centrifuge at 5000 $\mathrm{rpm}$ for $5 \mathrm{~min}$ and were resuspended with methaonl. The shell precusors were syntheized by $\mathrm{Nd}\left(\mathrm{CH}_{3} \mathrm{CO}_{2}\right)_{3}(0.48 \mathrm{mmol})$, $\mathrm{Yb}\left(\mathrm{CH}_{3} \mathrm{CO}_{2}\right)_{3}(0.48 \mathrm{mmol})$, and $\mathrm{Y}\left(\mathrm{CH}_{3} \mathrm{CO}_{2}\right)_{3}(1.28 \mathrm{mmol})$ as well as the process of $\mathrm{NaYF}_{4}: \mathrm{Yb} / \mathrm{Tm}$ synthesis. The core $\mathrm{NaYF}_{4}: \mathrm{Yb} / \mathrm{Tm}$ particles were embedded in $\mathrm{NaYF}_{4}: \mathrm{Yb} / \mathrm{Nd}$ nanoparticles in methanol with $\mathrm{NH}_{4} \mathrm{~F}(0.15 \mathrm{~g})$ and $\mathrm{NaOH}(0.1$ g). The reaction was performed at $290{ }^{\circ} \mathrm{C}$ for $2 \mathrm{~h}$, and the core-shell upconversion nanoparticles were obtained in cyclohexane after ethanol washing.

Preparation of UCNP@p-QD. The core-shell types of UCNPs consisting of $\mathrm{NaYF}_{4}: \mathrm{Yb} / \mathrm{Tm}$ (core) and $\mathrm{NaYF}_{4}: \mathrm{Yb} / \mathrm{Nd}$ (shell) were synthesized by the modified coprecipitation method. ${ }^{36}$ The UCNPs were then coated with a silica shed and surface modified with an amino group. The MMP2sensitive peptide was conjugated to UCNP through the amino group. The core/shell quantum dot that consists of $\mathrm{CuInS}_{2}$ (core) and $\mathrm{ZnS}$ (shell) was prepared by a modified hydrothermal method. ${ }^{23}$ UCNP@p was conjugated with the CIS/ $\mathrm{ZnS}$ QD in DMSO/chloroform solution (1:5) for $24 \mathrm{~h}$ conjugation because of disulfide binding. ${ }^{35}$ The folic acid (10 $\mathrm{nM}$ ) was further conjugated onto UCNP@p-QD using the NHS/EDC method. The upconversion nanoparticles were coated with silica shed using a modified microemulsion method. The water-soluble $\mathrm{NaYF}_{4}: \mathrm{Yb} / \mathrm{Tm} @ \mathrm{NaYF}_{4}: \mathrm{Yb} / \mathrm{Nd}$ particles were reconsitiued by alcohol-diluted $\mathrm{HCl}$ washing that removed the OA and ODE ligands and were dissolved in the mixture of IGEPAL CO-520 $(1 \mathrm{~mL})$ and cyclohexane $(10$ $\mathrm{mL}$ ) with vigorous stirring for $4 \mathrm{~h}$. Subsequently, the $\mathrm{pH}$ value was adjusted by $\mathrm{NH}_{4} \mathrm{OH}(0.1 \mathrm{~mL})$ solution, and the reaction was stirred for one additional hour. Finally, the tetraethyl orthosilicate (TEOS, 98\%, $30 \mu \mathrm{L}$ ) was slowly added into the reaction mixture by the speed of $40 \mu \mathrm{L}$ per hour for further 24 $\mathrm{h}$ stirring. The silica-coated nanoparticles were modified with an amino group by adding APTES $(0.1 \mathrm{~mL})$. The nanoparticles were collected by methanol precipation using centrifuging at $8000 \mathrm{rpm}$ for $20 \mathrm{~min}$. The MMP2-senstive peptide with a sequence of CSGAVRWLLTA was activated for $2 \mathrm{~h}$ by a mass proporation of peptide, NHS, and EDC of 5:1.5:1, respectively, in DMSO. Active peptides were grafted onto the amino group of silica-coated nanoparticles by the NHS/EDC method to form the peptide-grafted upconversion nanoparticle (UCNP@ p). The UCNP@p was dissolved in DMSO. To manifest the FRET process, UCNP@p $(0.5 \mathrm{mg} / \mathrm{mL})$ was conjugated with a different concentration of QD in DMSO/chloroform solution (5:1).

Surface Modification of UCNP@p-QD. CIS QD was synthesized using the DDT, on which the $\mathrm{ZnS}$ was coated to form a core/shell type nanoparticle. Since the DDT was hydrophobic, the CIS/ZnS QDs were dissolved in chloroform before use.UCNP@p-QD (100 mg) was PEGlyated using methyl-PEG 4 -thiol $(100 \mathrm{ng}$ ) and carboxy-PEG 12 -thiol (500 ng) in DMSO/chloroform solution $(1: 10)$ for $30 \mathrm{~min}$. The UCNP@p-QD was then washed by chloroform DMSO washing and was dissolved in $\mathrm{ddH}_{2} \mathrm{O}$. The photoluminescence of nanoparticles was detected by Fluoromax 3. The morphology was determined using the JEM-2100F (TEM, Japan) and JEOL transmission electron microscope (HRTEM, Japan). The electron gun of TEM and HRTEM worked at 80 and $200 \mathrm{keV}$, respectively. Zeta potential of conjugated nanocomposite was characterized at room temperature (RT) by a Malvern Zetasizer 3000.

Cell Culture. Oral cancer cell lines were cultured in a growth medium with 10\% FBS (Invitrogen, USA) and $1 \%$ penicillin-streptomycin-glutamine. The cell lines were cultured according to ATCC instruction. The cells were incubated in a $\mathrm{CO}_{2}$ incubator containing $5 \% \mathrm{CO}_{2}$ at $37{ }^{\circ} \mathrm{C}$. Cal27 and FADU were purchased from the ATCC cell line bank. The control vector (VC), pLAS3w.RFP-C.Ppuro, was purchased from RNAicore facility (Academia Sinica, Taiwan). Cal27/ MMP2 overexpressed MMP2 due to the lentiviral infection, at which the viruses were prepared by pLAS3W::MMP2.puro. Cal27/VC was introduced to the similar lentivirus DNA backbone that replaced the MMP2 reading frame with the frame of the red fluorescent protein (pLAS3w.RFP-C.Ppuro). The opposite Cal27/VC stable cell line expressed the RFP protein due to administration of the pLAS3w.RFP-C.Ppuroprepared lentivirus infection (RNAicore, Academia Sinica, Taiwan).

Cytoxicity Assay of UCNP@p-QD. The stable cell lines of $\mathrm{Cal27/VC}$ and Cal27/MMP2 were performed in MMP2 sensing. ${ }^{35}$ Cytotoxicity was evaluated by an alamar-blue assay using SpectraMax M2. ${ }^{37}$ An aliquot of 2000 cells was added in a 96-well plate. An aliquot of UCNP@p-QD was added with serial dilution $(250,83,27,9,3$, and $1 \mu \mathrm{g} / \mathrm{mL})$ for $72 \mathrm{~h}$ of incubation. The cytotoxic data were assessed from six independent tests with the standard deviation.

In Vitro and in Vivo MMP2 Detection. To perform the sensing ability of UCNP@p-QD, rhMMP2 was employed in 
dose- and time-dependent verification at $37^{\circ} \mathrm{C}$ incubation. The aliquot of rhMMP2 was added in $0.2 \mathrm{mg} / \mathrm{mL}$ of UCNP. In dose-dependent experiment, the rhMMP2 was serially diluted with $1,10^{-1}, 10^{-2}, 10^{-3}, 10^{-4}, 10^{-5}$, and $10^{-6} \mathrm{pg} / \mathrm{mL}$. The $10^{-3}$ $\mathrm{pg} / \mathrm{mL}$ of $\mathrm{rhMMP} 2$ was employed in a time-lapse FRET change. The time course of the FRET change was carried out with $0.2 \mathrm{mg} / \mathrm{mL}$ of UCNP@p-QD with $1 \mathrm{pg} / \mathrm{mL}$ of rhMMP2. To depict the FRET change, we focused on the fluorescence emission at 475 and $600 \mathrm{~nm}$, which were donated from UCNP and UCNP@p-QD, respectively. The data of three individual tests showed the means with standard derivation.

To explore in vivo MMP2 sensitivity of UCNP@p-QD, an MMP2-overexpressing stable cell line was employed compared to its cognate cell. Aliquot 20000 cells were seeded onto the coverslips for overnight incubation, and the UCNP@p-QD (10 $\mu \mathrm{g} / \mathrm{mL}$ ) was added for a further $12 \mathrm{~h}$ incubation. The cell and tissue images were obtained using a Leica TCS SP5 confocal microscope with multiphoton laser supplement.

Detection of MMP2 in the Mouse Xenograft Tumor Model. The animal experiments was approved by Academia Sinica Institutional Animal Care and Utilization Committee. The $5 \times 10^{6}$ aliquot of Cal27/VC and Cal27/MMP2 cells was subcutaneously injected on both opposite dorsal sides of the NSG mouse at 6-weeks old. ${ }^{38}$ After 4 weeks of tumor growth (62.5 $\mathrm{mm}^{3}$ of tumor volume), UCNP@p-QD $(20 \mathrm{mg} / \mathrm{mL})$ was administrated on both tumors by intratumoral injection. The real-time images were obtained using In-Vivo Xtreme (Bruker, Germany), with an additional $808 \mathrm{~nm}$ infrared diode laser module $(1.50 \mathrm{~W})$. The RFP acquisition was detected at $600 \mathrm{~nm}$ using $540 \mathrm{~nm}$ excitation. Xenograft tumors were harvested using a formalin-fixed and paraffin-embedded method. The tumor section was stained with propidium iodide $(1 \mu \mathrm{g} / \mathrm{mL})$ according to instruction. The images were obtained by confocal microscopy.

\section{ASSOCIATED CONTENT}

\section{S Supporting Information}

The Supporting Information is available free of charge on the ACS Publications website at DOI: 10.1021/acsomega.7b01494.

The characterizations of UCNP@p, UCNP@p-QD, conjugation of UCNP@p and CIS/ZnS QD, and FRET process are shown. The cell sensing and animal study are also disclosed (PDF)

\section{AUTHOR INFORMATION}

\section{Corresponding Authors}

*E-mail: dptsai@phys.ntu.edu.tw.

*E-mail: mhsiao@gate.sinica.edu.tw.

*E-mail: rsliu@ntu.edu.tw.

\section{ORCID}

Ru-Shi Liu: 0000-0002-1291-9052

Michael Hsiao: 0000-0001-8529-9213

Din Ping Tsai: 0000-0002-0883-9906

\section{Author Contributions}

Yung-Chieh Chan and Michael Hsiao designed and performed the study and cowrote the manuscript. Chieh-Wei Chen, MingHsien Chan, Ru-Shi Liu, and Din Ping Tsai provided technical support and materials. Michael Hsiao and Din Ping Tsai discussed the data and developed the theoretical aspect and wrote the manuscript. All authors commented on the manuscript.

\section{Notes}

The authors declare no competing financial interest.

\section{ACKNOWLEDGMENTS}

The authors acknowledge financial support from Ministry of Science and Technology, Taiwan (Grant Nos. MOST 1062745-M-002-003-ASP and MOST 104-2113-M-002-012-MY3) and Academia Sinica (Grant No. AS-103-TP-A06). They are also grateful to National Center for Theoretical Sciences, NEMS Research Center of National Taiwan University, National Center for High-Performance Computing, Taiwan, and Research Center for Applied Sciences, Academia Sinica, Taiwan, for their support. This research was also supported by Academia Sinica and Ministry of Science and Technology (MOST 106-0210-01-15-02, MOST 107-0210-01-19-01).

\section{ABBREVIATIONS}

MMP, matrix metalloproteinase; UCNP, upconversion nanoparticle; CIS $/ \mathrm{ZnS} \mathrm{QD}, \mathrm{CuInS}_{2} / \mathrm{ZnS}$ quantum dot; ECM, extracellular matrix; FRET, fluorescence resonance energy transfer; TEM, transmission electron microscope; EDS, energy-dispersive spectrometer; HNSCC, head-and-neck squamous-cell carcinoma

\section{REFERENCES}

(1) Ghajar, C. M.; Peinado, H.; Mori, H.; Matei, I. R.; Evason, K. J.; Brazier, H.; Almeida, D.; Koller, A.; Hajjar, K. A.; Stainier, D. Y.; Chen, E. I.; Lyden, D.; Bissell, M. J. The perivascular niche regulates breast tumour dormancy. Nat. Cell Biol. 2013, 15 (7), 807-17.

(2) Malanchi, I.; Santamaria-Martinez, A.; Susanto, E.; Peng, H.; Lehr, H. A.; Delaloye, J. F.; Huelsken, J. Interactions between cancer stem cells and their niche govern metastatic colonization. Nature 2012, 481 (7379), 85-9.

(3) Kessenbrock, K.; Plaks, V.; Werb, Z. Matrix metalloproteinases: regulators of the tumor microenvironment. Cell 2010, 141 (1), 52-67.

(4) Chen, Y. S.; Meng, F.; Li, H. L.; Liu, Q. H.; Hou, P. F.; Bai, J.; Zheng, J. N. Dicer suppresses MMP-2-mediated invasion and VEGFAinduced angiogenesis and serves as a promising prognostic biomarker in human clear cell renal cell carcinoma. Oncotarget 2016, 7 (51), 84299-84313.

(5) Detry, B.; Erpicum, C.; Paupert, J.; Blacher, S.; Maillard, C.; Bruyere, F.; Pendeville, H.; Remacle, T.; Lambert, V.; Balsat, C.; Ormenese, S.; Lamaye, F.; Janssens, E.; Moons, L.; Cataldo, D.; Kridelka, F.; Carmeliet, P.; Thiry, M.; Foidart, J. M.; Struman, I.; Noel, A. Matrix metalloproteinase-2 governs lymphatic vessel formation as an interstitial collagenase. Blood 2012, 119 (21), 5048-56.

(6) Jana, S.; Chatterjee, K.; Ray, A. K.; DasMahapatra, P.; Swarnakar, S. Regulation of Matrix Metalloproteinase-2 Activity by COX-2-PGE2pAKT Axis Promotes Angiogenesis in Endometriosis. PLoS One 2016, 11 (10), e0163540.

(7) Jafarian, A. H.; Vazife Mostaan, L.; Mohammadian Roshan, N.; Khazaeni, K.; Parsazad, S.; Gilan, H. Relationship between the Expression of Matrix Metalloproteinase and Clinicopathologic Features in Oral Squamous Cell Carcinoma. Iranian J. Otorhinolaryngology 2015, 27 (80), 219-23.

(8) Badiga, A. V.; Chetty, C.; Kesanakurti, D.; Are, D.; Gujrati, M.; Klopfenstein, J. D.; Dinh, D. H.; Rao, J. S. MMP-2 siRNA inhibits radiation-enhanced invasiveness in glioma cells. PLoS One 2011, 6 (6), e20614.

(9) Zhu, L.; Wang, T.; Perche, F.; Taigind, A.; Torchilin, V. P. Enhanced anticancer activity of nanopreparation containing an MMP2-sensitive PEG-drug conjugate and cell-penetrating moiety. Proc. Natl. Acad. Sci. U. S. A. 2013, 110 (42), 17047-52.

(10) Yang, Y.; Yue, C.; Han, Y.; Zhang, W.; He, A.; Zhang, C.; Yin, T.; Zhang, Q.; Zhang, J.; Yang, Y.; Ni, J.; Sun, J.; Cui, D. TumorResponsive Small Molecule Self-Assembled Nanosystem for Simulta- 
neous Fluorescence Imaging and Chemotherapy of Lung Cancer. Adv. Funct. Mater. 2016, 26 (47), 8735-8745.

(11) Hou, W.; Xia, F.; Alves, C. S.; Qian, X.; Yang, Y.; Cui, D. MMP2-Targeting and Redox-Responsive PEGylated Chlorin e6 Nanoparticles for Cancer Near-Infrared Imaging and Photodynamic Therapy. ACS Appl. Mater. Interfaces 2016, 8 (2), 1447-57.

(12) Lin, C. W.; Chou, Y. E.; Chiou, H. L.; Chen, M. K.; Yang, W. E.; Hsieh, M. J.; Yang, S. F. Pterostilbene suppresses oral cancer cell invasion by inhibiting MMP-2 expression. Expert Opin. Ther. Targets 2014, 18 (10), 1109-20.

(13) Panth, K. M.; van den Beucken, T.; Biemans, R.; Lieuwes, N. G.; Weber, M.; Losen, M.; Yaromina, A.; Dubois, L. J.; Lambin, P. In vivo optical imaging of MMP2 immuno protein antibody: tumor uptake is associated with MMP2 activity. Sci. Rep. 2016, 6, 22198.

(14) Wu, S.; Butt, H. J. Near-Infrared-Sensitive Materials Based on Upconverting Nanoparticles. Adv. Mater. 2016, 28 (6), 1208-26.

(15) Gargas, D. J.; Chan, E. M.; Ostrowski, A. D.; Aloni, S.; Altoe, M. V.; Barnard, E. S.; Sanii, B.; Urban, J. J.; Milliron, D. J.; Cohen, B. E.; Schuck, P. J. Engineering bright sub-10-nm upconverting nanocrystals for single-molecule imaging. Nat. Nanotechnol. 2014, 9 (4), 300-5.

(16) Ai, F.; Ju, Q.; Zhang, X.; Chen, X.; Wang, F.; Zhu, G. A coreshell-shell nanoplatform upconverting near-infrared light at $808 \mathrm{~nm}$ for luminescence imaging and photodynamic therapy of cancer. Sci. Rep. 2015, 5, 10785.

(17) Wilhelm, S.; Hirsch, T.; Patterson, W. M.; Scheucher, E.; Mayr, T.; Wolfbeis, O. S. Multicolor upconversion nanoparticles for protein conjugation. Theranostics 2013, 3 (4), 239-48.

(18) Li, Y.; Tang, J.; He, L.; Liu, Y.; Liu, Y.; Chen, C.; Tang, Z. CoreShell Upconversion Nanoparticle@Metal-Organic Framework Nanoprobes for Luminescent/Magnetic Dual-Mode Targeted Imaging. Adv. Mater. 2015, 27 (27), 4075-80.

(19) Yao, C.; Wang, P.; Li, X.; Hu, X.; Hou, J.; Wang, L.; Zhang, F. Near-Infrared-Triggered Azobenzene-Liposome/Upconversion Nanoparticle Hybrid Vesicles for Remotely Controlled Drug Delivery to Overcome Cancer Multidrug Resistance. Adv. Mater. 2016, 28 (42), 9341-9348.

(20) Li, X.; Deng, D.; Xue, J.; Qu, L.; Achilefu, S.; Gu, Y. Quantum dots based molecular beacons for in vitro and in vivo detection of MMP-2 on tumor. Biosens. Bioelectron. 2014, 61, 512-8.

(21) Li, T.-L.; Teng, H. Solution synthesis of high-quality CuInS2 quantum dots as sensitizers for $\mathrm{TiO} 2$ photoelectrodes. J. Mater. Chem. 2010, 20 (18), 3656-3664.

(22) Xie, R.; Rutherford, M.; Peng, X. Formation of High-Quality IIII-VI Semiconductor Nanocrystals by Tuning Relative Reactivity of Cationic Precursors. J. Am. Chem. Soc. 2009, 131 (15), 5691-5697.

(23) Chen, C.-W.; Wu, D.-Y.; Chan, Y.-C.; Lin, C. C.; Chung, P.-H.; Hsiao, M.; Liu, R.-S. Evaluations of the Chemical Stability and Cytotoxicity of CuInS2 and CuInS2/ZnS Core/Shell Quantum Dots. J. Phys. Chem. C 2015, 119 (5), 2852-2860.

(24) Foda, M. F.; Huang, L.; Shao, F.; Han, H. Y. Biocompatible and highly luminescent near-infrared $\mathrm{CuInS}(2) / \mathrm{ZnS}$ quantum dots embedded silica beads for cancer cell imaging. ACS Appl. Mater. Interfaces 2014, 6 (3), 2011-7.

(25) Fan, W.; Huang, P.; Chen, X. Overcoming the Achilles' heel of photodynamic therapy. Chem. Soc. Rev. 2016, 45 (23), 6488-6519.

(26) Bashkatov, A. N.; Genina, E. A.; Kochubey, V. I.; Tuchin, V. V. Optical properties of human skin, subcutaneous and mucous tissues in the wavelength range from 400 to $2000 \mathrm{~nm}$. J. Phys. D: Appl. Phys. 2005, 38 (15), 2543-2555.

(27) Hudson, D. E.; Hudson, D. O.; Wininger, J. M.; Richardson, B. D. Penetration of laser light at 808 and $980 \mathrm{~nm}$ in bovine tissue samples. Photomed. Laser Surg. 2013, 31 (4), 163-8.

(28) Zhan, Q.; Qian, J.; Liang, H.; Somesfalean, G.; Wang, D.; He, S.; Zhang, Z.; Andersson-Engels, S. Using 915 nm Laser Excited Tm3+/ Er3+/Ho3+-Doped NaYbF4 Upconversion Nanoparticles for in Vitro and Deeper in Vivo Bioimaging without Overheating Irradiation. ACS Nano 2011, 5 (5), 3744-3757.

(29) Chen, C.-W.; Lee, P.-H.; Chan, Y.-C.; Hsiao, M.; Chen, C.-H.; Wu, P. C.; Wu, P. R.; Tsai, D. P.; Tu, D.; Chen, X.; Liu, R.-S. Plasmon- induced hyperthermia: hybrid upconversion $\mathrm{NaYF} 4: \mathrm{Yb} / \mathrm{Er}$ and gold nanomaterials for oral cancer photothermal therapy. J. Mater. Chem. B 2015, 3 (42), 8293-8302.

(30) Tamang, S.; Beaune, G.; Texier, I.; Reiss, P. Aqueous phase transfer of $\mathrm{InP} / \mathrm{ZnS}$ nanocrystals conserving fluorescence and high colloidal stability. ACS Nano 2011, 5 (12), 9392-402.

(31) Gu, Z.; Yan, L.; Tian, G.; Li, S.; Chai, Z.; Zhao, Y. Recent advances in design and fabrication of upconversion nanoparticles and their safe theranostic applications. Adv. Mater. 2013, 25 (28), 375879.

(32) Lin, P. C.; Chou, P. H.; Chen, S. H.; Liao, H. K.; Wang, K. Y.; Chen, Y. J.; Lin, C. C. Ethylene glycol-protected magnetic nanoparticles for a multiplexed immunoassay in human plasma. Small 2006, 2 (4), 485-9.

(33) Park, J.; Kim, S.-W. CuInS2/ZnS core/shell quantum dots by cation exchange and their blue-shifted photoluminescence. J. Mater. Chem. 2011, 21 (11), 3745-3750.

(34) Ryu, E.; Kim, S.; Jang, E.; Jun, S.; Jang, H.; Kim, B.; Kim, S.-W. Step-Wise Synthesis of InP/ZnS Core-Shell Quantum Dots and the Role of Zinc Acetate. Chem. Mater. 2009, 21 (4), 573-575.

(35) Chan, Y. C.; Chen, C. W.; Chan, M. H.; Chang, Y. C.; Chang, W. M.; Chi, L. H.; Yu, H. M.; Lin, Y. F.; Tsai, D. P.; Liu, R. S.; Hsiao, M. MMP2-sensing up-conversion nanoparticle for fluorescence biosensing in head and neck cancer cells. Biosens. Bioelectron. 2016, $80,131-9$.

(36) Guan, M.; Dong, H.; Ge, J.; Chen, D.; Sun, L.; Li, S.; Wang, C.; Yan, C.; Wang, P.; Shu, C. Multifunctional upconversion-nanoparticles-trismethylpyridylporphyrin-fullerene nanocomposite: a nearinfrared light-triggered theranostic platform for imaging-guided photodynamic therapy. NPG Asia Mater. 2015, 7, e205.

(37) Hedberg, Y. S.; Herting, G.; Latvala, S.; Elihn, K.; Karlsson, H. L.; Odnevall Wallinder, I. Surface passivity largely governs the bioaccessibility of nickel-based powder particles at human exposure conditions. Regul. Toxicol. Pharmacol. 2016, 81, 162-170.

(38) Shultz, L. D.; Schweitzer, P. A.; Christianson, S. W.; Gott, B.; Schweitzer, I. B.; Tennent, B.; McKenna, S.; Mobraaten, L.; Rajan, T. V.; Greiner, D. L.; et al. Multiple defects in innate and adaptive immunologic function in NOD/LtSz-scid mice. J. Immunology (Baltimore, Md.: 1950) 1995, 154 (1), 180-91. 